\title{
Expanding the Role of Neuromodulation for Overactive Bladder: New Indications and Alternatives to Delivery
}

\author{
Ngoc-Bich Le $\cdot$ Ja-Hong Kim
}

Published online: 25 November 2010

(C) The Author(s) 2010. This article is published with open access at Springerlink.com

\begin{abstract}
From the time that it was granted US Food and Drug Administration approval, neuromodulation has secured a firm position in the treatment algorithm for overactive bladder. With neuromodulation, physicians were able to bridge the gap between the two ends of the treatment spectrum (medical therapy and open surgery). Sacral nerve stimulation has been the most widely used form of neuromodulation. Recent modifications to its design, namely the development of the tined lead and the launching of the refined InterStim II (Medtronic, Minneapolis, MN), have made sacral nerve simulation even less invasive and more effective. While InterStim is maintaining a level of success with these advancements, peripheral means of neuromodulation are being explored. The current literature takes a closer look at posterior tibial and pudendal nerve stimulation as alternatives to sacral nerve stimulation. The field of neuromodulation is also expanding in terms of the target patient population, as it is being used to treat children, patients with neurological disease, and others. As the role of neuromodulation increases, we must continue to assess its efficacy, safety, and cost-effectiveness in comparison to other therapeutic options.
\end{abstract}

Keywords Overactive bladder Sacral neuromodulation . Urinary incontinence $\cdot$ Neurostimulation $\cdot$ Sacral nerve stimulation · InterStim · Posterior tibial nerve stimulation .

N.-B. Le $\cdot$ J.-H. Kim $(\bowtie)$

Division of Pelvic Medicine and Reconstructive Surgery, Department of Urology, David Geffen School of Medicine at UCLA, Frank Clark Urology Center,

200 Medical Plaza, Suite 140,

Los Angeles, CA 90025, USA

e-mail: jhkim@mednet.ucla.edu
Peripheral nerve stimulation · Efficacy · Cost-effectiveness · Safety $\cdot$ Pudendal nerve stimulation $\cdot$ Neurogenic bladder . Cardiac pacemaker

\section{Introduction}

Overactive bladder $(\mathrm{OAB})$ is defined as urgency with or without urgency incontinence, usually with frequency ( $>8$ micturitions/24 h) and nocturia [1]. Conservative management of $\mathrm{OAB}$ usually includes pelvic floor physical therapy and/or use of pharmacotherapy such as antimuscarinic medications. However, a significant number of patients are refractory to pharmacotherapy, and long-term compliance is poor, so much so that only $30 \%$ of individuals with $\mathrm{OAB}$ syndrome still take their medication 1 year after initiation [2]. Traditionally, patients who failed conservative measures were left with highly invasive treatment options, such as myomectomy, augmentation cystoplasty, and various urinary diversions [3]. In 1997, the US Food and Drug Administration approved neuromodulation for urgency incontinence, and subsequently for urgency-frequency syndrome and nonobstructive chronic urinary retention. Although its mode of action is still not completely understood, recent research indicates that this therapy involves not just electrostimulation of sacral nerves but also neuromodulation due to somatosensory bladder afferents projecting into the pontine micturition center in the brainstem [4]. The objective of this article is to review the current literature and discuss the latest advances, significant trends, and current debates in the treatment of $\mathrm{OAB}$ with neuromodulation. Of particular interest are the articles focusing on peripheral nerve stimulation (PNS), cost-effectiveness, and nontraditional patient populations. 


\section{Cost-Effectiveness, Adverse Effects, and Safety}

During the past year, several articles examined the efficacy and cost-effectiveness of neuromodulation, as well as the adverse effects and safety profile of this procedure. In one such article, Siddiqui et al. [5•] systematically reviewed the literature regarding the efficacy of sacral nerve stimulation (SNS) using the InterStim (Medtronic, Minneapolis, MN) for women with refractory $\mathrm{OAB}$. The highest-quality data were found in three independent studies of efficacy. In these studies, incontinent episodes per day and pad usage significantly decreased after SNS therapy. After SNS, there was a significant decrease in mean incontinent episodes per day (2-3) and mean daily pad use (1-3). About $45 \%$ of patients reported "cure," or lack of daily incontinence episodes, up to 3 years after implant. Moreover, $54 \%$ of patients maintained improvements in daily incontinence episodes after implant. Subjective outcomes were also assessed and shown to be beneficial. Amundsen et al. [6] reported a 65- to 67-point decrease in Incontinence Impact Questionnaire (IIQ) scores 2 years after SNS implant.

In a separate article, Siddiqui et al. [7••] compared the costeffectiveness of SNS versus that of intravesical botulinum A toxin (BoNT-A) for treatment of refractory urge incontinence. Using the Markov decision model to compare cost (2008 US dollars) and effectiveness (quality-adjusted lifeyears [QALYs]), they calculated the incremental costeffectiveness ratio (ICER), which compares the difference in cost with the difference in effectiveness (ie, QALYs). In the best-case scenario, SNS was more expensive (\$15,743 vs $\$ 4,392)$ and more effective (1.73 vs 1.63 QALYs) than BoNT-A during a 2-year period. During a 2-year period, the ICER was $\$ 116,427$ per QALY, indicating that although SNS was more effective, BoNT-A was the more cost-effective option. One could argue that the cost-effectiveness comparison could change dramatically if it were recalculated beyond the 2-year period. However, the authors were limited to a 2year period because long-term data regarding BoNT-A injections are limited.

In addition to demonstrating that this procedure is effective, the literature also suggests that neuromodulation is relatively safe. Six good studies were available regarding adverse events associated with SNS [5•]. Adverse events mainly encompassed pain, infection, and change in clinical effect, often due to lead migration. The surgical revision rates when using tined leads ranged from $3 \%$ to $16 \%$, as most complications were managed conservatively. Six percent of patients were explanted due to lack of efficacy, and $5 \%$ to $11 \%$ were explanted due to infection.

Another safety issue regarding sacral neuromodulation is the potential for cross-talk between the InterStim device and cardiac pacemakers. Roth [8] recently pre- sented a series of three patients with cardiac pacemakers who subsequently underwent staged SNS implantation, and two patients who had successful internal pulse generator (IPG) implantation who later required cardiac pacemaker implantation. Patients had continuous cardiac monitoring by anesthesia, and there were no observed changes in rhythm perioperatively. This case series includes the two patients who did not have their IPGs turned off at the time of cardiac pacemaker implantation. At the time of 2-week follow-up, interrogation of the IPGs revealed that the settings had remained the same on both devices. The IPGs were all programmed for continuous bipolar stimulation. They were implanted on the contralateral side of the cardiac pacemakers in the three patients who had previously undergone cardiac pacemaker placement. Of note, none of the pacemakers had cardioversion or defibrillator capabilities. Based on this study, SNS can be safely used in patients with cardiac pacemakers.

\section{Alternatives to Sacral Nerve Stimulation}

\section{Posterior Tibial Nerve Stimulation}

During the past year, the bulk of the literature regarding advancements in neuromodulation for OAB has focused on alternatives to SNS. The literature suggests that PNS via the posterior tibial nerve or the pudendal nerve may be viable options. Posterior tibial nerve stimulation (PTNS) was first introduced by McGuire and colleagues in 1983, and more recent studies have confirmed a $60 \%$ to $80 \%$ positive response rate after 10 to 12 weekly treatments [9]. PTNS is performed by inserting a 34-gauge needle about 3 to $4 \mathrm{~cm}$ cephalad to the medial malleous, between the posterior margin of the tibia and soleus muscle. Once connected, an adjustable voltage pulse intensity of 0 to $10 \mathrm{~mA}$, a fixed pulse width of 200, and a frequency of $20 \mathrm{~Hz}$ were delivered during weekly 30-min sessions. Correct position is confirmed by flexion of the great toe or fanning of the toes and a tingling sensation. Adverse events associated with PTNS are minimal, mostly minor hematomas at the site of needle insertion.

Peters et al. [10॰] published a randomized, multicenter study that compared the efficacy of PTNS with that of extended-release tolterodine, also known as the OrBIT (Overactive Bladder Innovative Therapy) trial. A total of 100 adults with urinary frequency were randomly assigned to 12 weeks of treatment with PTNS or $4 \mathrm{mg} / \mathrm{d}$ of extendedrelease tolterodine. After 12 weeks of therapy, the PTNS arm reported a $79.5 \%$ cure or improvement rate, compared with $54.8 \%$ of those on tolterodine $(P=0.01)$ on the global response assessments. The two groups had similar improvements on objective measures, including reductions in 
urinary frequency, urge urinary incontinence episodes, urge severity, and nighttime voids, and increased voided volume. The authors concluded that PTNS was safe and efficacious, with statistically significant subjective and objective improvements comparable to that of pharmacotherapy.

Peters et al. [11•] also compared the efficacy of PTNS to that of sham in a multicenter, double-blind, randomized trial known as SUmiT (Study of Urgent PC Versus Sham Effectiveness in Treatment of Overactive Bladder Symptoms). After 12 weeks, 54.5\% of the PTNS patients reported moderate or markedly improved response from baseline, compared with $20.9 \%$ of sham patients $(P<$ $0.001)$. PTNS also increased voided volume when compared with baseline from 83 to $169.5 \mathrm{~mL}(P=0.01)$. Patients were not able to correctly identify if they were part of the sham or PTNS group, as both groups had leads placed. The results from this study suggest that the therapeutic effect of PTNS is a result of stimulation of the posterior tibial nerve and not a placebo effect.

Following the encouraging results from the SUmiT and OrBIT studies demonstrating true therapeutic effects comparable to those of tolterodine, other authors focused on various treatment courses and durability. The traditional PTNS treatment course runs for 12 weeks, with weekly 30min sessions. Yoong et al. [12] reported initial outcome data following a shortened 6-week treatment protocol with PTNS. Forty-three women underwent this shortened treatment course and were evaluated with bladder symptom diaries and the Incontinence Impact Questionnaire (IIQ-7), which is a health-related quality-of-life questionnaire. A positive response to therapy was defined as 1) OAB symptoms no longer being bothersome, 2) reduction by half in frequency episodes, and 3 ) reduction by $25 \%$ in IIQ7 outcomes. The positive response rate was $69.7 \%$, with an almost $50 \%$ decrease in the median daytime and nocturnal frequency ( 11.8 vs 6.9 and 3.5 vs $1.8 ; P<0.05)$. Patients reported fewer urge incontinence episodes per $24 \mathrm{~h}$, from a median of 3.5 to $2.4(P<0.05)$. The median IIQ-7 scores decreased by $25 \%$ (30.4 vs $24.3 ; P<0.05)$, and the median number of pads used in 24 hours decreased by $34 \%$ (3.8 vs $2.5 ; P<0.05)$. However, the participants reported return of symptoms 3 weeks after the treatment, and no predictors of duration of symptomatic relief could be identified. These early data suggest that the PTNS treatment course can be halved, but more conclusive studies are needed to determine the optimal duration of initial treatment and maintenance program.

The question of durability of PTNS treatment was addressed by MacDiarmid et al. [13•] in the second phase of the OrBIT study. Thirty-three of the 35 responders from the initial 12-week treatment course elected for maintenance therapy. Participants selected treatment intervals allowing them to control OAB symptoms at an acceptable level. A total of 32 completed 6 months of maintenance, and 25 completed 12 months. The participants received a mean of $12.1 \pm 4.9$ treatments over an average of 263 days, with a mean of 21 days (median, 17) between treatments. However, the participants had longer time intervals during the second 6 months than during the first 6 months of the evaluation (14 vs 24 days). Subjective global assessments showed a sustained improvement of symptoms from 12 weeks at the 6-month and 12-month evaluations (94\% and $96 \%$, respectively). There were also objective improvements from baseline, with decreases of 2.8 voids per day $(P<0.001), 1.6$ urge incontinence episodes per day $(P<0.001), 0.8$ nocturnal voids $(P<0.05)$, and an increase of $39 \mathrm{~mL}$ in voided volume $(P<0.01)$. The authors concluded that PTNS can be a viable long-term therapeutic option for $\mathrm{OAB}$ patients.

A global evaluation of PTNS as a treatment option for OAB was performed by Ridout and Yoong [9] with a review of the published literature. Six articles were identified that assessed the efficacy of PTNS as therapy for OAB. These studies used various criteria for "positive response," such as objective reduction in symptoms and the request for maintenance therapy following completion of the 12-week course. Using these varying definitions of success, PTNS has been reported to have a $60 \%$ to $81 \%$ response rate. Most authors agree that PTNS requires indefinite maintenance therapy, but the specifics of the maintenance program have not yet been defined. Van der Pal et al. [14] showed that $64 \%$ of patients reported greater than $50 \%$ worsening in frequency and incontinence episodes after a 6-week pause in treatment. Overall, PTNS seems to offer symptomatic relief for OAB patients who are refractory to conservative therapy, but standard guidelines for initial therapy and maintenance regimens are lacking.

\section{Pudendal Neuromodulation}

In addition to the sacral and the posterior tibial nerves, physicians can target the pudendal nerves for neuromodulation. The sacral nerve sensory afferent fibers originate in the pudendal nerve, which innervates the pelvic floor muscles, external urethral and anal sphincters, and pelvic organs. Peters et al. [15] performed a retrospective review of patients undergoing tined lead placement at the pudendal nerve via the ischial-rectal approach for chronic pudendal neuromodulation. There were 84 patients with various primary urologic diagnoses, including interstitial cystitis/ painful bladder syndrome, urgency/frequency or urge incontinence, nonobstructive urinary retention, and pelvic/ bladder pain without interstitial cystitis. Almost all individuals with a history of failed sacral neuromodulation responded to the pudendal lead stimulation (93.2\% [41 of 44]). Overall, positive pudendal response $(\geq 50 \%$ improvement on the 
pudendal lead) was achieved in 60 of 84 participants (71.4\%). These are encouraging results that warrant further investigation and suggest that pudendal nerve-targeted neuromodulation may be a viable alternative to SNS.

\section{Technological Development}

Since its introduction, Medtronic has made some advancement in the design of the SNS device. The introduction of the tined lead 5 years ago changed sacral neuromodulation from a single-stage open surgery requiring general anesthesia to a two-stage, minimally invasive, percutaneous procedure with local anesthesia. Because there is less lead migration, the permanent tined lead allows for a longer trial period, during which the physician and patient can reprogram the device until they achieve optimal settings. As a result of this extended test period, the technical failure rate has decreased, and the response rate has almost doubled to approximately $80 \%$ [3].

The most recent technological development in the InterStim apparatus has been the InterStim II device. The InterStim II received its regulatory approval in 2006. Like its prototype, the InterStim II is an implantable neurostimulator but is $50 \%$ lighter and smaller. This change purportedly increases patient comfort and makes the implant procedure easier and quicker for the surgeon [3]. A postmarket, Web-based survey among 11 European experts in urology, gynecology, and gastroenterology evaluated their initial experience with the new InterStim II therapy in 55 patients [16]. The less bulky InterStim II implantable neurostimulator allowed for a tinier incision and shallower pocket $(3.5 \mathrm{~cm}$ less), which made implantation more tolerable, especially for thinner patients. Moreover, the new device does not require an extension cable, which reduced operative time. The new small iCon patient programmer also offers the patient more flexibility, with four preset programs to optimize the response rate.

\section{Novel Applications}

During the past year, several authors reported their experience with sacral neuromodulation in nontraditional patient populations, including patients with neurogenic detrusor overactivity, failed augmentation cystoplasty, and children. A recent article from Italy looked at PTNS as a treatment option for children with lower urinary tract dysfunction [17]. Forty-four children with a variety of urologic diagnoses (including idiopathic $\mathrm{OAB}$, dysfunctional voiding, underactive bladder, underactive valve bladder, and neurogenic bladder) were enrolled in a prospective clinical trial of PTNS efficacy. A total of 25 were female and 18 were male (average age, $10.7 \pm 4.8$ years). They underwent the conventional 12-week course of PTNS with weekly 30-min sessions. Follow-up assessment was performed every 6 months for 2 years after treatment. Symptomatic improvement was greater in non-neurogenic than in neurogenic cases $(78 \%$ vs $14 \% ; P<0.002)$. At 1 -year followup, the cure rate was greater in dysfunctional voiding than in OAB cases $(71 \%$ vs $41 \%)$, and it remained the same at 2 years. Chronic neuromodulation was required to maintain results in $29 \%$ of patients with dysfunctional voiding and $50 \%$ of patients with OAB. Thus, PTNS appears to be a viable option for treating pediatric lower urinary tract dysfunction, especially in non-neurogenic cases.

As in the pediatric population, lower urinary tract dysfunction is also common in adults with neurological disease. However, apart from spinal cord injury patients, neuromodulation is not commonly offered to this group of patients. Van Rey and Heesakkers [18•] wrote a review article on the applications of neurostimulation for urinary storage and voiding dysfunction in neurological patients. This article reviewed the use and results of pudendal nerve stimulation, SNS, and lower limb stimulation in patients with neurogenic detrusor overactivity. Some evidence has demonstrated SNS efficacy in treating neurogenic bladder disorders in multiple sclerosis patients, but this population has a chance of negative test stimulation that is four times higher [18•]. Pudendal nerve stimulation via dorsal penile/clitoral nerve stimulation has been shown to benefit spinal cord injury patients by increasing cystometric capacity and inhibiting neurogenic bladder overactivity [19]. The current use of pudendal nerve stimulation is limited by a lack of reliable stimulation technique, as pudendal nerve stimulation via tined leads and surface electrodes is still in the preliminary stages. As a third alternative to sacral and pudendal nerve stimulation, PTNS also has been reported to be effective for a patient population of individuals with multiple sclerosis, myelitis, incomplete spinal cord injury, stroke, and Parkinson's disease [20,21]. These studies suggest there is significant benefit to neuromodulation among this special patient population that warrants further study.

Part of the advantage of SNS is that it provides treatment for refractory $\mathrm{OAB}$ patients without requiring extensive surgery (eg, augmentation cystoplasty). However, some patients have already undergone augmentation cystoplasty and continue to suffer with OAB symptoms. Rasmussen et al. [21] reported on their case series of two such patients who were treated with sacral neuromodulation. One patient continued to void every 30 to $60 \mathrm{~min}$ despite medical therapy, intravesical treatment with BoNT-A, and augmentation cystoplasty. This patient ultimately underwent staged sacral approach implantation and was able to wait $3 \mathrm{~h}$ between voids during the trial period. She had durable improvement at 14 months of follow-up after the secondstage implantation. The second patient had undergone a supratrigonal ileocystoplasty but still voided every $1.5 \mathrm{~h}$ 
with volumes ranging from 50 to $220 \mathrm{~mL}$. After her staged sacral neuromodulator implantation, she was able to wait 3 to $4 \mathrm{~h}$, and her voided volume ranged from 300 to $500 \mathrm{~mL}$. Her improvements have persisted with a follow-up of 17 months. Both patients had videourodynamics as part of their preoperative assessment, which demonstrated good compliance and capacity. This case series indicates that SNS may be an important adjunctive therapy for patients with truly refractory $\mathrm{OAB}$ despite bladder augmentation.

\section{Conclusions}

During the past year, the literature regarding neuromodulation for treating $\mathrm{OAB}$ has focused heavily on validating PTNS and pudendal nerve stimulation as reasonable alternatives to SNS. Other significant articles re-examined SNS in terms of safety, efficacy, and cost-effectiveness. The general conclusion is that neuromodulation is an efficacious and safe treatment option for patients with medically refractory $\mathrm{OAB}$. The inclusion criteria for SNS treatment have expanded to embrace the neurogenic and pediatric populations, as well as those who have undergone augmentation cystoplasty. Future efforts should focus on defining optimal treatment and maintenance schedules for less invasive PTNS and reporting the preliminary experience with the new InterStim II device. The current developments in technology and clinical applicability will continue to broaden the field of neuromodulation for urologic patients.

Disclosure Dr. Kim has served as a consultant for American Medical Systems and has received honoraria from Pfizer. Dr. Le reported no potential conflicts of interest relevant to this article.

Open Access This article is distributed under the terms of the Creative Commons Attribution Noncommercial License which permits any noncommercial use, distribution, and reproduction in any medium, provided the original author(s) and source are credited.

\section{References}

Papers of particular interest, published recently, have been highlighted as:

- Of importance

-. Of major importance

1. Abrams P, Cardozo L, Fall M, et al.: The standardization of terminology of lower urinary tract function: Report from Standardisation Subcommittee of the International Continence Society. Neurourol Urodyn 2002; 21:167-178.
2. Kelleher CJ, Cardozo LD, Khullar V, et al.: A medium term analysis of the subjective efficacy of treatment for women with detrusor instability and low bladder. British Journal of Obstetrics and Gynaecology 1997, 104: 988-993.

3. Spinelli M, Sievert KD.: Latest technologic and surgical developments in using InterStim therapy for sacral neuromodulation: Impact on treatment success and safety. Eur Urol 2008, 54: 1287-96.

4. Leng WW, Chancellor MB: How sacral nerve stimulation neuromodulation works. Urol Clin North Am 2005; 32: 11-8.

5. - Siddiqui NY, Wu JM, Amundsen CL: Efficacy and adverse events of sacral nerve stimulation for overactive bladder: A systematic review. Neurourol Urodyn 2010, 29:S18-S23. This is a timely review of the available literature on SNS that contributes to the evidence-based approach to treating refractory $O A B$.

6. Amundsen CL, Romero AA, Jamison MG, et al.: Sacral neuromodulation for intractable urge incontinence: Are there factors associated with cure? Urology 2005;66: 746-50.

7. - Siddiqui NY, Amundsen CL, Visco AG, et al.: Costeffectiveness of sacral neuromodulation versus intravesical botulinum A toxin for treatment of refractory urge incontinence. J Urol 2009; 182:2799-804. Cost-effectiveness, especially in terms of medical treatment, is an integral issue in the contemporary discussions on health care reform and the economy.

8. Roth TM: Sacral neuromodulation and cardiac pacemakers. Int Urogynecol J 2010;21:1035-1037.

9. Ridout AE, Yoong W: Tibial nerve stimulation for overactive bladder syndrome unresponsive to medical therapy. Int Urogynecol J Pelvic Floor Dysfunct 2010; 21: 1035.

10. • Peters KM, MacDiarmid SA, Wooldridge LS et al.: Randomized trial of percutaneous tibial nerve stimulation versus extendedrelease tolterodine: results from the overactive bladder innovative therapy trial. J Urol 2009; 178: 2029. This is a laudable effort to generate level I evidence in this field.

11. - Peters KM, Carrico DJ, Perez-Marrero RA, et al.: Randomized trial of percutaneous tibial nerve stimulation versus Sham efficacy in the treatment of overactive bladder syndrome: results from the SUmiT trial. J Urol 2010, 183:1438-43. This is another laudable effort to generate level I evidence in this field.

12. Yoong W, Ridout AE, Damodaram M, et al.: Neuromodulative treatment with percutaneous tibial nerve stimulation for intractable detrusor instability: outcomes following a shortened 6-week protocol. BJU Int 2010 Jun 29 (Epub ahead of print).

13. - MacDiarmid SA, Peters KM, Shobeiri SA, et al.: Long-term durability of percutaneous tibial nerve stimulation for the treatment of overactive bladder. J Urol 2010; 183: 234. This article addresses a key concern surrounding the use of PTNS in the treatment of $O A B$.

14. van der Pal F, van Balken MR, Heesakkers JP, et al.: Percutaneous tibial nerve stimulation in the treatment of refractory overactive bladder syndrome: Is maintenance treatment necessary? British Journal of Urology International 2006, 97:547-560.

15. Peters KM, Killinger KA, Boguslawski BM, et al.: Chronic pudendal neurostimulation: Expanding available treatment options for refractory urologic symptoms. Neurourol Urodyn 2001, 29:1267-1271.

16. Sievert KD, Matzel K, Ratto C, et al.: Permanente sakrale Neuromodulation mittels InterStim : Ergebnisse einer Anwenderbefragung zu aktuellen technischen Entwicklungen. J Urol Urogynakol 2007, 14:14-6.

17. Capitanucci ML, Camanni D, Demelas F, et al.: Long-term efficacy of percutaneous tibial nerve stimulation for different types of lower urinary tract dysfunction in children. J Urol 2009, 182: 2056. 
18. - Van Rey FS, Heesakkers JP: Applications of neurostimulation for urinary storage and voiding dysfunction in neurological patients. Urol Int 2009; 81: 373. This article raises the possibility of using neuromodulation to treat voiding dysfunction in patients with neurological disorders.

19. Scheepens WA, Jongen MM, Nieman FH, et al.: Predictive factors for sacral neuromodulation in chronic lower tract dysfunction. Urology 2002, 60:598-602.
20. Finazzi AE, Parisi A, Petta F, et al.: Percutaneous tibial nerve stimulation in the treatment of lower urinary tract dysfunction secondary to multiple sclerosis. Paper presented at the meeting of the International Continence Society. Florence, Italy; 2003.

21. Rasmussen NT, Guralnick ML, O'Connor RC: Successful use of sacral neuromodulation after failed bladder augmentation. Can Urol Assoc J 2009, 3:E49-E50. 